\title{
Cuidados paliativos e Ensino Médico: Revisão sistemática
}

\author{
Palliative care and Medical Education: Systematic review \\ Cuidados paliativos y Educación Médica: Revisión sistemática
}

Recebido: 14/01/2021 | Revisado: 17/01/2021 | Aceito: 21/01/2021 | Publicado: 25/01/2021

\author{
Andrea Augusta Castro \\ ORCID: https://orcid.org/0000-0001-5887-0314 \\ Universidade do Estado do Rio de Janeiro, Brasil \\ E-mail: castro.andreaaugusta@gmail.com \\ Stella Regina Taquette \\ ORCID: https://orcid.org/0000-0001-7388-3025 \\ Universidade do Estado do Rio de Janeiro, Brasil \\ E-mail: stella.taquette@gmail.com \\ Caio Assunção Rocha Pereira \\ ORCID: https://orcid.org/0000-0002-2239-865X \\ Universidade do Estado do Rio de Janeiro, Brasil \\ E-mail: caio.assuncaop@gmail.com
}

\begin{abstract}
Resumo
Introdução: Cuidados paliativos (CP) estão no quotidiano dos serviços de saúde, face ao aumento da carga das doenças crônicas, do envelhecimento e incorporação tecnológica na saúde. Há consenso sobre a importância do ensino em CP na graduação, entretanto muitas escolas não o praticam. Esta revisão objetivou sintetizar o conhecimento produzido em estudos científicos sobre ensino em CP na graduação em medicina e seu impacto na formação médica. Métodos: Foi realizada revisão sistemática de estudos publicados no PubMed, com os descritores: "medical education" OR "curriculum"; "palliative care" AND "clinical competence". Foram encontrados 89 artigos, excluídos 67 títulos e resumos e 22 artigos foram analisados. Resultados: A leitura dos artigos deu origem a três categorias temáticas. Na primeira categoria, elenca-se abordagem biopsicossocial e espiritual, cuidado centrado na pessoa e família, comunicação, trabalho interprofissional, e manejo de sintomas como competências essenciais. Na segunda, os desafios e estratégias apontados foram o escasso tempo curricular no ensino em CP, a falta de contexto clínico para o aprendizado reflexivo, o déficit de docentes preparados e a sua ausência formal nos currículos. O impacto do ensino em $\mathrm{CP}$ na aprendizagem na graduação refere-se a ser catalisador do desenvolvimento profissional e propiciar competências emocionais aos futuros médicos. Conclusão: Os estudos analisados demonstram benefícios do ensino em CP na formação médica, através de um ensino longitudinal, uma vez que potencializam a aquisição de competências essenciais no exercício da medicina e em especial no trato de pessoas portadoras de doenças ameaçadoras à vida.
\end{abstract}

Palavras-chave: Cuidados paliativos; Educação médica; Competências; Graduação.

\begin{abstract}
Introduction: Palliative care underlies the daily life of health services, due to the increased burden of chronic diseases and aging. While teaching is fundamental, there are significant gaps in the medical curriculum. There is a consensus about the relevance of teaching in undergraduate courses; however, many schools do not include it in the curriculum. Methods: The search strategy was used, and descriptors were defined as follows: "palliative care" AND "medical education" OR "curriculum and competence clinical", total of 89 papers were identified. From this sample, 67 papers were not included and 22 papers analyzed. Results: The emerging categories were as follows: impact on learning by the incorporation of palliative care education in the undergraduate medical course, essential competencies in palliative care in the medical undergraduate course and obstacles and paths aimed at its insertion in the undergraduate medical curriculum. Disciplinary modules contribute to changes in the approach to finitude and the acquisition of emotional competencies by professionals. This paper addresses a systematic review of how and why palliative care teaching should be carried out in graduation, considering difficulties and challenges for curricular incorporation. The main obstacles identified were lack of clinical context for education, lack of curricular time in the face of the demand, low support and structure, lack of qualified teachers, fragmented teaching and formal absence in curricula. Conclusion: The studies analyzed demonstrate benefits of teaching in PC in medical training, through longitudinal education, since they enhance the acquisition of essential skills in the exercise of medicine and especially in the treatment of people with lifethreatening diseases.
\end{abstract}

Keywords: Palliative care; Medical education; Clinical competence; Graduation.

\section{Resumen}

Introducción: Los cuidados paliativos (PC) se encuentran en la vida cotidiana de los servicios de salud, habida cuenta de la mayor carga de enfermedades crónicas, el envejecimiento y la incorporación tecnológica en la salud. Hay consenso sobre la importancia de la enseñanza en PC en los estudios de pregrado, sin embargo muchas escuelas no lo practican. 
Esta revisión tenía como objetivo sintetizar los conocimientos producidos en estudios científicos sobre la enseñanza de PC en la medicina de pregrado y su impacto en la formación médica. Métodos: Se realizó una revisión sistemática de los estudios publicados en PubMed, con los descriptores: "educación médica" O "currículo";" paliativos" y "competencia clínica". Encontramos 89 artículos, 67 títulos y resúmenes fueron excluidos y se analizaron 22 artículos. Resultados: La lectura de los artículos dio lugar a tres categorías temáticas. En la primera categoría, se enumeran un enfoque biopsicosocial y espiritual, atención de persona y centrado en la familia, comunicación, trabajo interprofesional y manejo de síntomas como competencias esenciales. En el segundo, los desafíos y estrategias señalados fueron el escaso tiempo curricular en la enseñanza en PC, la falta de contexto clínico para el aprendizaje reflexivo, el déficit de los maestros preparados y su ausencia formal en los planes de estudio. El impacto de la enseñanza de CP en el aprendizaje de pregrado se refiere a ser un catalizador para el desarrollo profesional y proporcionar habilidades emocionales a los futuros médicos. Conclusión: Los estudios analizados demuestran los beneficios de la enseñanza en PC en la educación médica, a través de la educación longitudinal, ya que mejoran la adquisición de competencias esenciales en la práctica de la medicina y especialmente en el tratamiento de personas con enfermedades potencialmente mortales.

Palabras clave: Cuidados paliativos; Educación médica; Competencia clinical; Graduación.

\section{Introdução}

O envelhecimento populacional, o aumento da carga das doenças crônicas e a inserção tecnológica na saúde trouxeram implicações nas práticas médicas e no modelo de atenção. A organização dos CP é norteada pela constatação de que cada pessoa exigirá uma atenção específica, seja por alguns dias, meses ou anos (Murray, et al., 2015).

Cuidados paliativos (CP) estão indicados a serem praticados desde o diagnóstico de doenças ameaçadoras à vida e nos vários estágios do processo de adoecimento, incluindo investigação e abordagem para prevenir complicações. Visa aliviar o sofrimento e melhorar a qualidade de vida, disponibilizar apoio clínico, assim como abordar os problemas psicológicos e espirituais que cursam na progressão da doença, a um custo relativamente baixo, evitando tratamentos agressivos (WHO, 2002)

O ensino em CP na graduação, portanto, é uma necessidade atual para garantir a competência de futuros médicos neste campo de atuação. $\mathrm{O}$ aprendizado em CP possibilita reflexões sobre a morte e o morrer e tem sido considerado uma estratégia para a qualificação da atenção à saúde e à defesa dos direitos humanos (Gibbins, et al., 2009). Embora considerado essencial, observa-se que a maioria das escolas médicas não incluem o tema $\mathrm{CP}$ no currículo e os alunos relatam pouco conhecimento e treinamento na graduação (Chiu, et al., 2015).

O ensino em CP na graduação pode ser abordado em todas as áreas de atuação e especialidades médicas e despertar a atenção dos futuros médicos, podendo melhorar a qualidade de vida do paciente. É observado maior senso de controle na interação com pacientes e familiares, compaixão, empatia e respeito, através da exposição a pacientes portadores de doenças graves e reflexão sobre os aspectos da finitude (Crawford \& Zambrano, 2015). As iniciativas na área do ensino e aprendizagem em CP são muito distintas nos diversos países, tornando importante apontar as competências essenciais e os desafios a serem enfrentados no sentido de contribuir para o aprendizado do tema. Diante da relevância do ensino em CP levantam-se questões tais como: o que é prioritário ser ensinado e em que cenários deve se dar o aprendizado? Quais os desafios a serem enfrentados e estratégias apontadas para o ensino em CP? Quais transformações o ensino de CP promove na formação médica?

Esse estudo foi realizado com o objetivo de sintetizar o conhecimento científico produzido sobre ensino em CP com foco nos conteúdos, cenários de aprendizagem, desafios enfrentados e avanços observados. Pretende-se com os resultados oferecer subsídios às propostas curriculares de ensino em $\mathrm{CP}$.

\section{Métodos}

Foi realizada revisão sistemática de estudos publicados em periódicos indexados na base de dados do PubMed. Esta base de dados foi escolhida por agregar maior número de fontes bibliográficas de qualidade, reconhecidas internacionalmente. Foi utilizada a estratégia de busca através de descritores em três blocos, a saber: I- ((“Education, Medical”[mh] OR “Education, 
Medical"[tiab] OR "Medical Education"[tiab] OR "Medical Graduation"[tiab] OR "Curriculum"[tiab])); AND II) ("Palliative Care"[mh] OR "Palliative Care"[tiab] OR "Palliative Medicine"[tiab] OR "Care, Palliative"[tiab] OR “Medicine, Palliative"[tiab] AND III) "Clinical Competence". A busca foi filtrada nos últimos cinco anos (período de 2014 a 2020) e pesquisas realizados com seres humanos, de artigos, resumos e títulos com os descritores selecionados.

Foram encontrados 89 artigos. Após leitura de todos os títulos, 40 foram excluídos por não preencherem o critério de inclusão: abordar estudos sobre o ensino em CP na graduação em medicina. Em seguida foram lidos os resumos dos 49 títulos restantes. Após a leitura, 26 títulos foram excluídos: 18 por não abordarem CP especificamente, seis por tratarem de experiências educacionais não relativas à graduação, dois por serem de revisão bibliográfica e um por se referir a instrumento de avaliação do ensino. Restaram 32 artigos que foram lidos e analisados e dez eliminados pelos seguintes motivos: sete não tratavam temas educacionais, dois não estavam disponíveis na íntegra e um tratava de ensino em religião específica. Os 22 artigos restantes foram os incluídos nesta revisão e submetidos à análise de conteúdo temática. O fluxograma de seleção dos estudos pode ser visualizado na Figura 1.

Figura 1. Diagrama de busca dos artigos da revisão sistemática- 2020.

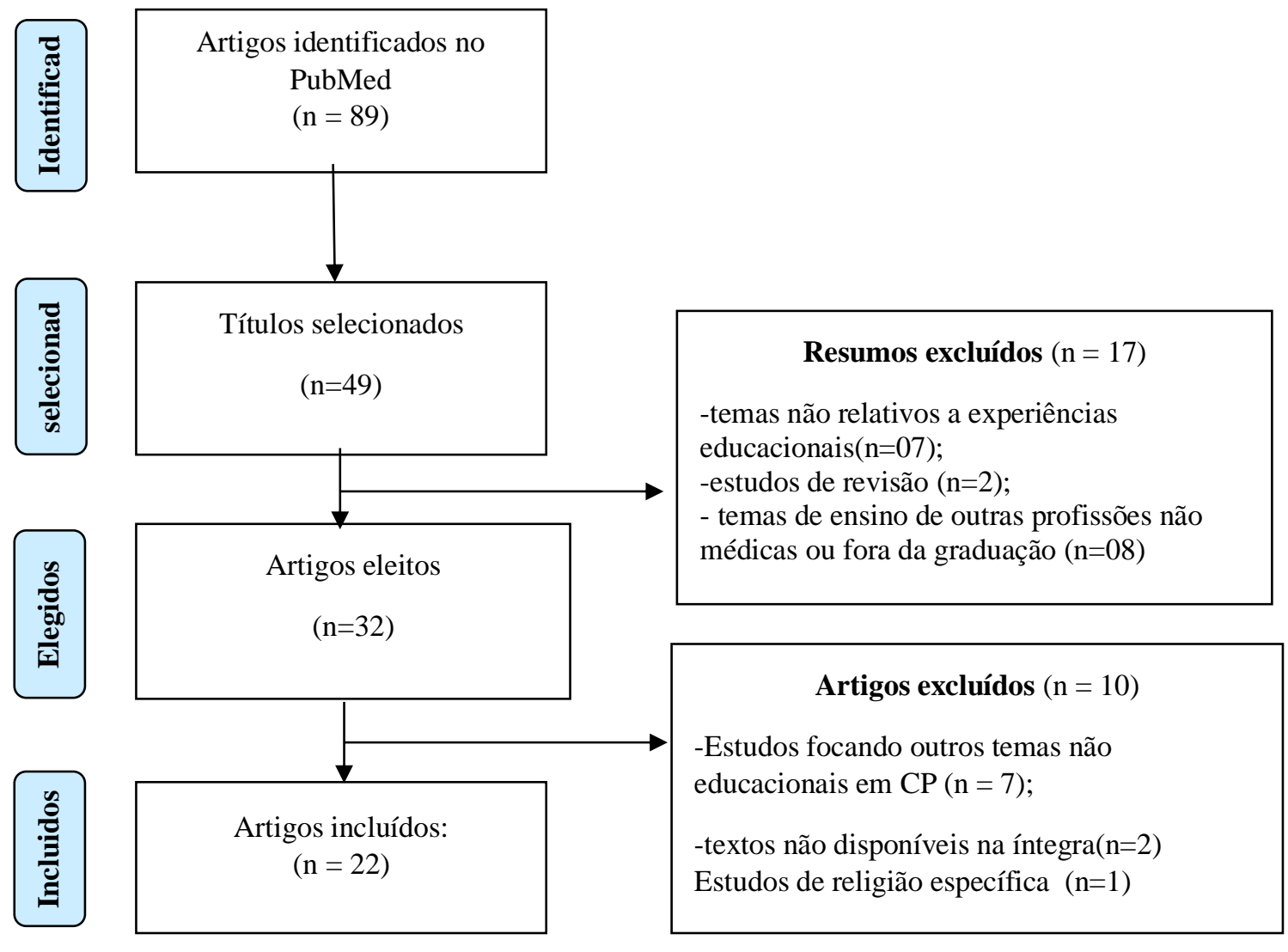

Fonte: Autores

No decorrer da análise do material bibliográfico foram incluídos mais 13 artigos publicados em periódicos científicos da área e documentos internacionais relativos aos temas para discussão dos dados. (Taquette \&Borges, 2020) 


\section{3-Resultados e Discussão}

A maior parte dos estudos levantados, 14 (63,7\%) é de natureza qualitativa, ou misto, do tipo pesquisa-ação, com intervenções educativas em CP. Quanto à distribuição geográfica continental, onze foram desenvolvidos na América do Norte, oito na Europa, um na América do Sul, um na Oceania e um na Ásia.

Após leitura e releitura dos artigos, no esforço de organizar os temas relativos ao ensino médico em CP e avanços proporcionados, os textos deram origem às seguintes categorias: I) competências consideradas essenciais em CP na graduação médica, II) desafios e avanços apontados para sua inserção no currículo médico da graduação e III) o impacto da inserção do ensino em CP na graduação médica considerando a aprendizagem dos estudantes.

As agências internacionais em CP recomendam a inclusão das suas competências gerais na graduação em medicina. Os países têm realidades muito distintas, refletindo a importância dos aspectos culturais, econômicos, políticos e sociais de cada escola. Embora seja considerado importante, existem desafios para a inserção do ensino em cuidados paliativos em todo o mundo (WHO, 2014).

$\mathrm{Na}$ Tabela 1 podem ser visualizados os artigos revisados sistematizados considerando local e ano da publicação, tipo de estudo, categoria classificada, objetivos, amostra dos participantes e principais resultados.

\section{I- As competências essenciais em CP na graduação médica}

Nesta categoria foram identificados seis estudos relacionados às competências essenciais em CP nos cursos de medicina. Evidenciou-se que as matrizes curriculares apresentadas são muito variadas, mesmo em países onde a medicina paliativa tornouse obrigatória nos currículos das escolas médicas (Smith, et al., 2019). Os principais tópicos identificados como competências foram: as habilidades de comunicação; o manejo de dor e sintomas; os aspectos psicossociais e espirituais; bioética e legislação; o papel do sistema de saúde; e equipe interdisciplinar e autocuidado. Para aquisição destas competências essenciais valoriza-se o trabalho em equipe interprofissional e colaborativo (Pastrana, et al, 2016).

Os objetivos de aprendizagem do ensino em CP na graduação não estão bem definidos. Existe fragmentação do ensino e ausência formal nos currículos para a aprendizagem no ensino de pacientes na finitude. O ensino em CP gerais deve abordar o manejo de sintomas físicos e psicológicos, necessidades espirituais, respeito às diretivas da vontade e o respeito às diferenças culturais. As temáticas de maior interesse dos estudantes são manejo nos últimos dias, comunicação para abordar preferências de tratamento e sintomas psicológicos (Carroll, et al.,2019).

Três domínios de aprendizagem predominaram nas propostas curriculares, no campo afetivo, cognitivo e psicomotor: maior respeito aos valores dos pacientes; compreensão do processo de morrer e adequação no uso de opioides (Schiessi, et al., 2013). As competências essenciais para estudantes, médicos e residentes em medicina da família e medicina interna são a comunicação e o manejo da dor e sintomas que estão contidas em cinco domínios de conhecimentos: comunicação e abordagem centrada na pessoa; habilidades no processo decisório no fim de vida; princípios e práticas dos cuidados paliativos; filosofia e papel do cuidado paliativo e hospice; e o controle da dor e outros sintomas (Spalding \& Yardley, 2016).

É relevante debater na graduação as questões relativas à morte e ao morrer e desenvolver habilidades de comunicação, trabalho em equipe e suporte à família (Schaeffer, et al.,2014). O autor destaca também conhecimentos sobre controle de sinais e sintomas e aspectos espirituais e religiosos, visando minimizar o sofrimento de quem enfrenta a fase de finitude da vida (Scheiderman, et al.,2011). A aproximação com pacientes terminais facilita a aquisição de habilidades de comunicação e despertam competências relacionais e atitudinais (Kriesen \& Müller, 2018).

A inclusão do ensino em CP na graduação pode favorecer ao estudante desenvolver competências para o cuidado ampliado ao paciente, não apenas na finitude, mas em todas as fases da vida da pessoa (Weber et al., 2016). A aproximação do 
futuro médico com pacientes em doenças avançadas pode desenvolver a aquisição de habilidades emocionais, e uma abordagem terapêutica ampliada nos cuidados gerais (Back et al., 2016).

Tabela 1. Relação dos artigos relacionados a competências nucleares em CP na graduação segundo autor, ano, local, tipo de estudo, objetivos, amostra e resultados.

\begin{tabular}{|c|c|c|c|}
\hline $\begin{array}{l}\text { Autor, ano, tipo de } \\
\text { pesquisa e } \\
\text { local }\end{array}$ & Objetivos & Amostra & Resultados e conclusão \\
\hline $\begin{array}{l}\text { Carroll T et all, } 2019 \\
\text { Estudo } \\
\text { qualitativo/entrevista } \\
\text { (NY, USA) }\end{array}$ & $\begin{array}{l}\text { Avaliar } \\
\text { congruências de } \\
\text { competências no } \\
\text { ensino em CP entre } \\
\text { os médicos } \\
\text { não paliativistas e } \\
\text { paliativistas }\end{array}$ & $\begin{array}{l}144 \text { médicos } \\
\text { não paliativistas e } 18 \\
\text { médicos paliativistas. }\end{array}$ & $\begin{array}{l}\text { Embora existam diferenças, as } \\
\text { preferenciais para ambos são: } \\
\text { manejo nos últimos dias, } \\
\text { sintomas psicológicos e } \\
\text { comunicação. }\end{array}$ \\
\hline $\begin{array}{l}\text { Kriesen U et all, } \\
2018 \\
\text { Estudo } \\
\text { qualitativo/entrevista } \\
\text { (Rostock,Alemanh) }\end{array}$ & $\begin{array}{l}\text { Analisar percepção } \\
\text { do ensino à beira do } \\
\text { leito na perspectiva } \\
\text { de estudantes, } \\
\text { funcionários e } \\
\text { pacientes }\end{array}$ & $\begin{array}{l}21 \text { estudantes, vinte } \\
\text { pacientes e } 19 \\
\text { membros da equipe } \\
\text { de } \mathrm{CP}\end{array}$ & $\begin{array}{l}\text { O contato com pacientes em } \\
\text { finitude é valorizado entre } \\
\text { estudantes e pacientes } \\
\text { favorecendo aquisição de } \\
\text { competências atitudinais. }\end{array}$ \\
\hline $\begin{array}{l}\text { Spalding at all, } 2016 \\
\text { Estudo qualitativo/ } \\
\text { grupos focais } \\
\text { (Staffordshire, UK }\end{array}$ & $\begin{array}{l}\text { Explorar a } \\
\text { percepção dos } \\
\text { estudantes no } \\
\text { ensino em CP em } \\
\text { diferentes cenários } \\
\text { de aprendizado com } \\
\text { crianças e seus } \\
\text { familiares em um } \\
\text { hospice }\end{array}$ & $\begin{array}{l}\text { Sete crianças, cinco } \\
\text { pais e seis } \\
\text { funcionários }\end{array}$ & $\begin{array}{l}\text { O estudo evidenciou maior } \\
\text { compreensão dos estudantes } \\
\text { nas habilidades de } \\
\text { comunicação, trabalho em } \\
\text { equipe e cuidado centrado na } \\
\text { pessoa. }\end{array}$ \\
\hline $\begin{array}{l}\text { Pastrana } T \text { et all } \\
\text { Estudo qualitativo/ } \\
\text { grupo focal } \\
\text { (Colômbia) } 2015\end{array}$ & $\begin{array}{l}\text { Descrever as etapas } \\
\text { e os resultados do } \\
\text { trabalho utilizado } \\
\text { na definição de } \\
\text { competências para } \\
\text { o ensino em CP no } \\
\text { país }\end{array}$ & $\begin{array}{l}36 \text { participantes em } \\
18 \text { universidades }\end{array}$ & $\begin{array}{l}\text { O trabalho definiu consenso } \\
\text { de competências em CP: } \\
\text { princípios, controle de } \\
\text { sintomas, cuidados em fim de } \\
\text { vida, questões éticas e legais, } \\
\text { abordagem biopsicossocial e } \\
\text { espiritual e trabalho em } \\
\text { equipe. }\end{array}$ \\
\hline $\begin{array}{l}\text { Smith et all, } 2018 \\
\text { Estudo } \\
\text { qualitativo/entrevista } \\
\text { (Lancaster, UK) }\end{array}$ & $\begin{array}{l}\text { Descrever o } \\
\text { desenvolvimento da } \\
\text { primeira estrutura } \\
\text { nacional do Reino } \\
\text { Unido para orientar } \\
\text { o ensino }\end{array}$ & $\begin{array}{l}33 \text { professores em } 33 \\
\text { escolas médicas }\end{array}$ & $\begin{array}{l}\text { Foram organizados oito } \\
\text { domínios-chave da prática em } \\
\text { cuidados intensivos em CP }\end{array}$ \\
\hline $\begin{array}{l}\text { Back et all, } 2016 \\
\text { Método } \\
\text { qualitativo/grupos } \\
\text { focais } \\
\text { (Seattle, USA) }\end{array}$ & $\begin{array}{l}\text { Avaliar programa } \\
\text { de desenvolvimento } \\
\text { de resiliência dos } \\
\text { alunos e clínicos na } \\
\text { prevenção de burno } \\
\text { ut em CP }\end{array}$ & $\begin{array}{l}\text { Equipes de CP, } \\
\text { estudantes, médicos, } \\
\text { gestores, psicólogos e } \\
\text { mentores. }\end{array}$ & $\begin{array}{l}\text { As habilidades de resiliência } \\
\text { são tão importantes quanto as } \\
\text { habilidades de comunicação e } \\
\text { gerenciamento de sintomas; é } \\
\text { influenciado por recursos } \\
\text { pessoais, demandas de } \\
\text { trabalho e autorreflexão. }\end{array}$ \\
\hline
\end{tabular}


Os achados dos estudos revelam um predomínio para a aquisição das competências atitudinais e relacionais. Assim como, observamos um corpo de competências nucleares bem delineadas, como o manejo de sintomas, comunicação, bioética, e abordagem biopsicossocial e espiritual. O ensino ao longo da formação pode preparar o futuro profissional para uma atuação qualificada e assertiva como requer o cuidado de pessoas portadoras de doenças ameaçadoras à vida.

\section{II- Principais desafios e avanços no ensino em CP}

O ensino médico em CP e alguns avanços alcançados são tratados em seis artigos que abordam seus desafios e apontam estratégias importantes, conforme a Tabela 2. Em países como Suíça, Finlândia e Alemanha, a construção de diretrizes para a inserção do ensino em CP está em fase avançada, em outros o processo ainda enfrenta dificuldades, como na Colômbia e EUA.

Os autores relatam falta de contexto clínico para o aprendizado na finitude da vida, uma vez que no último ano do curso, a maioria dos estudantes revela falta de confiança e déficit de conhecimento em CP (Kriesen \& Müller, 2018). Embora a educação em medicina paliativa seja considerada importante, não está organizada no ambiente docente-assistencial. Entre barreiras na implementação do ensino são incluídas a falta de tempo, devido a outras demandas que recebem mais prioridade e o déficit de docentes preparados.

A aquisição do aprendizado em CP é oportunizada em diferentes cenários, tanto com crianças ou idosos portadores de doenças crônicas. Esse contato potencializa mudanças de atitudes a partir de vivências significativas. O principal desafio é a falta de exposição a estes cenários desde o início do curso, mantendo os alunos longe dos pacientes em final de vida, não oportunizando experiências variáveis e o desenvolvimento de habilidade bioética, a partir de reflexões sobre as vivências no aprendizado (Annear, et al., e 2016),

Uma das principais dificuldades na atenção de pessoas com doenças avançadas é o sofrimento emocional dos educandos. Contudo, as sucessivas aproximações com estes pacientes são consideradas oportunidades de aprendizado e prevenção de burnout nos futuros médicos. Estes devem contar com retaguarda de docentes experientes, possibilitando conforto através da reflexão sobre as experiências, possibilitando o desenvolvimento pessoal, a autorreflexão e resiliência (Vergo et al., 2017).

Os desafios do ensino em CP requerem um contato continuado, possibilitando a compreensão da integralidade do processo saúde-doença (Schillerstrom et al., 2012). O aprendizado é construído através de módulos sucessivos de famílias de situações vivenciadas ao longo da formação (Smith \& Schaefer, 2014). Algumas estratégias apontadas para driblar os desafios e dificuldades referem-se à incorporação de ensino virtual, assim como simulações para o treinamento de habilidades de comunicação em ambientes não reais (Brown \& Schulz-Quach, 2018).

A inclusão do ensino em CP ao longo do curso parece ser uma importante estratégia para o preparo dos futuros médicos. Desta forma, o aprendizado vai sendo promovido ao longo da formação. O ensino em CP no currículo da graduação permite a formação de médicos mais preparados para lidar com situações de dor e outros sintomas. (MacPherson, et al., 2014), A aquisição das competências essenciais é observada no ensino longitudinal e a inserção de uma disciplina alavanca o aprendizado, possibilitando a abordagem integral e no manejo de sintomas nas pessoas portadoras de doenças avançadas (Letho, et al., 2017). 
Tabela 2. Relação de artigos relacionados aos avanços e desafios na inserção do ensino em CP no currículo médico segundo autor, ano, tipo de pesquisa, local, objetivos, amostra e resultados.

\begin{tabular}{|c|c|c|c|}
\hline $\begin{array}{l}\text { Autor, ano, tipo de } \\
\text { pesquisa e } \\
\text { local }\end{array}$ & Objetivos & Amostra & Resultados \\
\hline $\begin{array}{l}\text { Hawkins et all, } 2016 \\
\text { Estudo qualitativo / } \\
\text { narrativas } \\
\text { (Hillview Road, UK }\end{array}$ & $\begin{array}{l}\text { Avaliar impacto do } \\
\text { ensino em CP na } \\
\text { exploração de } \\
\text { preferências para o } \\
\text { cuidado no fim da } \\
\text { vida, através de } \\
\text { simulação de alta } \\
\text { performance }\end{array}$ & $\begin{array}{l}\text { sete estudantes } \\
\text { de medicina do } \\
\text { último ano da } \\
\text { graduação; }\end{array}$ & $\begin{array}{l}\text { O grau de sofrimento do } \\
\text { estudante e seus pares pôde } \\
\text { ser identificado em cenários } \\
\text { de simulação de alta } \\
\text { fidelidade com pacientes em } \\
\text { final de vida, podendo ser um } \\
\text { complemento para o } \\
\text { treinamento de habilidades de } \\
\text { comunicação na graduação. }\end{array}$ \\
\hline $\begin{array}{l}\text { Annear et all, } 2016 \\
\text { Estudo misto quanti-quali } \\
\text { (Hobart, Australia) }\end{array}$ & $\begin{array}{l}\text { Avaliar a aquisição } \\
\text { de competências } \\
\text { em diferentes } \\
\text { cenários de } \\
\text { aprendizado }\end{array}$ & $\begin{array}{l}61 \text { alunos do } \\
\text { quinto ano de } \\
\text { medicina }\end{array}$ & $\begin{array}{l}\text { As interações com pacientes } \\
\text { idosos em asilos podem } \\
\text { melhorar as atitudes dos } \\
\text { estudantes de medicina em } \\
\text { relação manejo das } \\
\text { demências. }\end{array}$ \\
\hline $\begin{array}{l}\text { Weber et all, } 2016 \\
\text { Estudo } \\
\text { quantitativo/questionário on- } \\
\text { line } \\
\text { (Mainz, Alemanha) }\end{array}$ & $\begin{array}{l}\text { Avaliar o } \\
\text { conhecimento em } \\
\text { cuidados } \\
\text { paliativos no } \\
\text { último ano da } \\
\text { graduação }\end{array}$ & $\begin{array}{l}92 \text { estudantes } \\
\text { do último ano } \\
\text { do curso de } \\
\text { medicina }\end{array}$ & $\begin{array}{l}\text { A maioria dos estudantes } \\
\text { apresenta baixa confiança e } \\
\text { pouco conhecimento sobre CP } \\
\text { ao final da graduação, sendo o } \\
\text { ensino longitudinal relevante. }\end{array}$ \\
\hline $\begin{array}{l}\text { Samala RV et all, } 2019 \\
\text { Estudo qualitativo/narrativas } \\
\text { (Ohio, EUA) }\end{array}$ & $\begin{array}{l}\text { Descrever o } \\
\text { impacto do ensino } \\
\text { em CP na } \\
\text { aprendizagem dos } \\
\text { estudantes }\end{array}$ & $\begin{array}{l}29 \text { narrativas de } \\
\text { oito estudantes } \\
\text { de } \\
\text { medicina após } \\
\text { visitas } \\
\text { domiciliares }\end{array}$ & $\begin{array}{l}\text { O ensino em CP traz o } \\
\text { reconhecimento } \\
\text { sobre desafios da } \\
\text { atenção domiciliar, trabalho } \\
\text { em equipe, cuidado centrado } \\
\text { na pessoa e família e é } \\
\text { catalisador do crescimento } \\
\text { pessoal. }\end{array}$ \\
\hline $\begin{array}{l}\text { Brown et all, } 2016 \\
\text { Estudo quantitativo- ensaio } \\
\text { clínico randomizado } \\
\text { (Seattle USA) }\end{array}$ & $\begin{array}{l}\text { Analisar o impacto } \\
\text { do ensino em CP } \\
\text { nas habilidades de } \\
\text { comunicação } \\
\text { através de } \\
\text { simulação }\end{array}$ & $\begin{array}{l}472 \text { internos de } \\
\text { medicina e } \\
\text { enfermagem }\end{array}$ & $\begin{array}{l}\text { O treinamento em } \\
\text { comunicação baseado em } \\
\text { simulação (Codetalk) foi } \\
\text { associado a aquisição de } \\
\text { competência nas habilidades } \\
\text { de comunicação. }\end{array}$ \\
\hline $\begin{array}{l}\text { Schulz-Quach et all, } 2018 \\
\text { Estudo quantitativo de corte } \\
\text { transversal } \\
\text { (Witten, Germany) }\end{array}$ & $\begin{array}{l}\text { Investigar impacto } \\
\text { na aquisição de } \\
\text { competências em } \\
\text { CP através do } \\
\text { ensino virtual }\end{array}$ & $\begin{array}{l}670 \text { estudantes } \\
\text { de medicina }\end{array}$ & $\begin{array}{l}\text { Outras abordagens, além do } \\
\text { ensino virtual são necessárias } \\
\text { para aquisição de } \\
\text { competências relacionais com } \\
\text { paciente e familiares e } \\
\text { aumento da autoestima dos } \\
\text { estudantes. }\end{array}$ \\
\hline
\end{tabular}

Fonte: Pubmed.

Os estudos revelam fragilidades emocionais e dificuldades na comunicação como desafios a serem superados pelas sucessivas aproximações aos pacientes portadores de doenças avançadas.

III - Impacto da inclusão do ensino em cuidados paliativo na graduação 
Nesta categoria foram abarcados dez estudos que apresentam as transformações ocorridas após a inclusão do ensino em CP na graduação em medicina, conforme relacionados na Tabela 3. Os estudos demonstram que o ensino em CP possibilita aquisição de competências importantes para os futuros médicos, principalmente no campo pessoal e emocional. Entretanto, é necessário um sistema avaliativo para seu aprimoramento e persistem os desafios na construção das melhores estratégias para o ensino em CP (Eychmüller et al., 2015).

As mudanças no futuro médico foram identificadas, principalmente no desenvolvimento de competências emocionais, relacionais, assim como na autorreflexão que pode potencializar a resiliência para o cuidado compassivo, incluindo a abordagem da espiritualidade. Quando ocorre a inclusão da disciplina $\mathrm{CP}$, é observado melhor desempenho e confiança nos alunos que tiveram esse espaço na sua formação (Ellman, et al., 2016),

A inclusão do ensino em CP na educação médica possibilita promover uma atitude consciente nos futuros médicos frente à eminência da morte (Taquette \& Rego, 2005) . É relevante abordar o afastamento que o morrer provoca, ajudando o estudante a vencer os medos e sofrimentos, possibilitando mais conforto nas sucessivas aproximações aos pacientes portadores de doenças avançadas (Romotzky et al., 2015)

O ensino em CP melhora a escala de eficácia em domínios importantes como comunicação e trabalho colaborativo e interdisciplinar, possibilitando aquisições de competências para os cuidados gerais (Saylor, et al.,2016 \& Peh, et al.,2017). O desenvolvimento de habilidades de comunicação pode ser promovido pelo ensino em $\mathrm{CP}$, como, por exemplo, ao explorar as preferências do paciente para o cuidado no fim da vida; na iniciação de conversação sobre questões espirituais e suas práticas, entre outros (Parick et al., 2017). Mudanças de valores e atitudes, assim como aquisição de habilidades de comunicação no ensino em CP foram demonstradas no processo avaliativo de estudantes (Von Gunten et al., 2017).

$\mathrm{O}$ ensino em CP contribui na superação de lacunas educacionais, e potencializa a integralidade da abordagem ao paciente e familiares, além de possibilitar maior colaboração com outros membros da equipe de saúde (Westcott et al., 2019). Os estudantes e pacientes valorizam o treinamento em CP na formação do médico, favorecendo a aquisição de competências atitudinais e de autorreflexão, podendo catalisar o crescimento pessoal do futuro médico (Samala et al., 2019).

Tabela 3. Artigos relacionados ao impacto nos estudantes frente a inserção do ensino em CP na graduação segundo autor, ano, tipo de estudo, local, objetivos, amostra e resultados.

\begin{tabular}{llll}
\hline $\begin{array}{l}\text { Autor, ano, tipo de } \\
\text { pesquisa e } \\
\text { local }\end{array}$ & Objetivos & Amostra & Resultados \\
\hline
\end{tabular}

Eychmüller et all, 2015

Estudo qualitativo/entrevista ( Berna,Suíça)

Parikh PP et all, 2016

Estudo quantitativo/

prospectivo

(Ohio, EUA)

Lehto et all, 2017

Estudo quanti e qualitativo

(Tempere/ Finlândia)

Avaliar
o monitoramento
longitudinal do
currículo nas
escolas médicas em
CP.

Avaliar o aprendizado das habilidades de comunicações no ensino em CP

\section{Analisar os} resultados da inserção do ensino longitudinal em CP e disciplina

$\begin{array}{ll}\text { cinco reitores } & \text { Reconhecimento da medicina } \\ \text { das faculdades } & \text { paliativa integrante ao } \\ \text { de medicina. } & \text { treinamento médico. }\end{array}$
$\begin{array}{ll}\text { das faculdades } & \text { paliativa integrante a } \\ \text { de medicina. } & \text { treinamento médico. }\end{array}$

69 estudantes O desenvolvimento de de habilidades de comunicações medicina no em CP é observado em $70 \%$ dos terceiro ano alunos da graduação

$80 \%$ de presença da turma, não há informação do quantitativo
Ao longo dos anos estudados, foi observada melhora da performance e aquisições de competências em CP. 
Vergo et all, 2016

Estudo quantitativo prospectivo

(New Hampshire, EUA)

Von Gunten et all, 2016

Estudo quantitativo/ corte transversal

(San Diego, EUA)

Ellman et all,2016

Estudo misto prospectivo quanti-

qualitativo(Connecticut, USA)

Saylor et all, 2016

Estudo quantitativo prospectivo randomizado (Newark, EUA)

Romotzky et all, 2014 Estudo qualitativo/entrevista (Cologne, Alemanha)

Ying Peh,

2016

Estudo quantitativo retrospectivo

(Singapore,Singapore)

Westcott AM et all,2018
Estudo qualitativo/entrevistas
(Hershey, USA)

Estudo qualitativo/entrevistas
Avaliar a aquisição de habilidades de comunicação em cuidados paliativos primários

Examinar o impacto do ensino em CP

Avaliar o impacto do ensino em CP em currículo longitudinal integrado
12 estudantes de medicina interna

448 internos de medicina

estudantes do quarto ano e 121 do terceiro ano

Os estudantes mostraram melhorias estatisticamente significativas na habilidade de comunicação geral e na resposta a emoções.

A aquisição de conhecimentos aumenta a confiança para a prática clínica dos estudantes e estes demonstram maiores preocupações éticas.

Os estudantes demonstraram aquisição de competências emocionais, atitudinais, de autorreflexão e apreciação de valores nos aspectos culturais e espirituais, trabalho interprofissional, melhora na comunicação, aceitação da morte e cuidado compassivo.

$\begin{array}{lll}\text { Aferir aquisição de } & 104 & \text { Houve melhora na competência } \\ \text { competências } & \text { estudantes de } & \text { interprofissional, na } \\ \text { relacionais e } & \text { enfermagem, } & \text { autoeficácia, nas atitudes das } \\ \text { competências } & \text { medicina e } & \text { relações entre médico e outras } \\ \text { interprofissionais } & \text { outras áreas } & \text { profissões }\end{array}$

Avaliar o ensino do $\mathrm{CP}$ com ênfase em habilidades de comunicação através de simulação Avaliar aquisição de competências após exposição ao ensino em CP da saúde. 17 estudantes de medicina

Os resultados indicam uma necessidade de treinamento em comunicação para estudantes de medicina, sendo a simulação uma alternativa.

Avaliar o impacto do ensino interprofissional no ensino médico em $\mathrm{CP}$
71 estudantes de medicina interna

$\begin{array}{ll}\text { dois capelães, } & \text { A participação da equipe } \\ \text { duas } & \text { multiprofissional contribui na } \\ \text { enfermeiras, } & \text { superação das lacunas } \\ \text { uma assistente } & \text { educacionais no ensino em CP. } \\ \text { social e um } & \text { entre as quais o cuidado } \\ \text { médico } & \begin{array}{l}\text { integral, comunicação e sentido } \\ \text { de colaboração entre os } \\ \text { assistente }\end{array} \\ & \text { profissionais. }\end{array}$

O ensino de um mês em CP possibilita maior confiança aos estudantes, particularmente na comunicação em final de vida.

Fonte: Pubmed.

A aquisição das competências em CP proporciona transformações que podem ser estendidas aos cuidados gerais nos demais ciclos de vida, e não apenas restrito ao período de finitude da vida.

\section{Conclusão}

A análise dos estudos revisados aponta a importância da inserção do ensino em CP na graduação para a aquisição de competências consideradas essenciais no exercício da medicina. Demonstra o impacto do ensino nos futuros profissionais, sendo 
uma oportunidade de aprendizagem nos domínios de comunicação, medicina centrada na pessoa e reflexão sobre os aspectos bioéticos. Reforça a necessidade deste ensino tanto por especialistas como por não especialistas neste campo.

Torna-se crucial, que as instituições acadêmicas formem profissionais capacitados a trabalharem em $\mathrm{CP}$ para o atendimento das demandas de saúde atuais resultantes do envelhecimento populacional, do aumento da carga das doenças crônicas e da inserção das novas tecnologias na saúde.

A presente revisão bibliográfica, apesar de limitada à base de dados do PubMed, serve como importante balizamento da recomendação da inclusão dos CP nos currículos atuais dos cursos de graduação em medicina.

Sugerimos trabalhos futuros que possam aprofundar o impacto do ensino em $\mathrm{CP}$, considerando o respeito cultural e as especificidades regionais, assim como as questões da bioética vivenciadas no cotidiano dos estudantes e profissionais de saúde.

\section{Referências}

Annear, M. J., Lea, E., Lo, A., Tierney, L., \& Robinson, A. (2016). Encountering aged care: a mixed methods investigation of medical students' clinical placement experiences. BMC Geriatr;16-38. 10.1186/s12877-016-0211-8.

Back, A. L., Steinhauser, K. E., Kamal, A. H., \& Jackson, V. A. (2016). Building Resilience for Palliative Care Clinicians: An Approach to Burnout. Prevention Based on Individual Skills and Workplace Factors. J Pain Symptom Manage. Aug;52(2):284-91. 10.1016/j.jpainsymman.2016.02.002

Brown, C. E., Back, A. L., Ford, D. W., Kross, E. K., Downey, L., Shannon, S. E., Curtis, J. R., \& Engelberg, R. A. (2018). Self-Assessment Scores Improve After Simulation-Based Palliative Care Communication Skill Workshops. Am J Hosp Palliat Care. Jan;35(1):45-51. 10.3928/01484834-20180720-08

Carroll, T., El-Sourady, M., Karlekar, M., \& Richeson, A. (2019). Primary Palliative Care Education Programs: Review and Characterization. Am J Hosp Palliat Care. Jun;36(6):546-49. 10.1177/1049909118809947.

Crawford, G. B., \& Zambrano, S. C.(2015). Junior doctors' views of how their undergraduate clinical electives in palliative care influenced their current practice of medicine Acad Med; 90(3): 338-44. 10.1097/ACM.0000000000000632

Ellman, M. S., Fortin, A. H., Putnam, A., \& Bia, M. (2016). Implementing and Evaluating a Four-Year Integrated End-of-Life Care Curriculum for Medical Students. Teach Learn Med.28(2):229-39. 10.1080/10401334.2016.1146601

Eychmüller, S., Forster, M., Gudat, H., Lütolf, U. M., \& Borasio, G. D. (2015) Undergraduate palliative care teaching in Swiss medical faculties: a nationwide survey and improved learning objectives.Med Educ. 2015 Nov 27;15:213. 10.1186/s12909-015-0485-0

Gibbins, J., McCoubrie, R.; Maher, J., \& Forbes, K.(2009). Incorporating palliative care into undergraduate curricula: lessons for curriculum development Med Educ; 43(8): 776-83. 10.1111/j.1365-2923.2009.03400.x

Kriesen, U., Altiner, A., \& Müller-Hilke, B. (2018). Perception of bedside teaching within the palliative care setting-views from patients, students and staff members. Ann Palliat Med. Oct;7(4):411-19. 10.21037/apm.2018.05.01

Lehto, J. T., Hakkarainen, K., Kellokumpu-Lehtinen, P. L., \& Saarto, T. (2017). Undergraduate curriculum in palliative medicine at Tampere University increases students' knowledge. BMC Palliat Care. Jan 25;16(1):13. 10.1186/s12904-016-0182-8

MacPherson, A., Lawrie, I., Collin, S., \& Forman, L. (2014).Teaching the difficult-to-teach topics BMJ Support Palliat Care;,4(1): 87-91. 10.1136/bmjspcare2012-000408

Murray, S. A., Firth, A., Schneider, N., Van den Eynde, B., Gomez-Batiste, X., Brogard T., et al. (2015). Promoting palliative care in the community: production of the primary palliative care toolkit by the European Association of Palliative Care Taskforce in primary palliative care. Palliat Med;29(2):101-11. https://eapcnet.wordpress.com/2015/02/11/toolkit-for-the-development-of-palliative-care-in-the-community/

Pastrana, T., Wenk, R., \& De Lima, L. (2016). Consensus-Based Palliative Care Competencies for Undergraduate Nurses and Physicians: A Demonstrative Process with Colombian Universities. J Palliat MedJan;19(1):76-82. 10.1089/jpm.2015.0202

Parikh, P. P., White, M. T., Buckingham, L., \& Tchorz, K. M.(2017) Evaluation of palliative care training and skills retention by medical students.J Surg Res; 211: 172-77. 10.1016/j.jss.2016.11.006

Peh, T. Y.,Yang, G. M., Krishna, L. K., \& Yee, A. C. (2017).Do Doctors Gain More Confidence from a Longer Palliative Medicine Posting? J Palliat Med. Feb;20(2):141-46. 10.1089/jpm.2016.0293

Romotzky, V., Galushko, M., Düsterdiek, A., Obliers, R., Albus, C., Ostgathe, C., \& Voltz, R. (2015)."It's Not that Easy"--Medical Students' Fears and Barriers in End-of-Life Communication.J Cancer Educ; 30(2): 333-39. 10.1007/s13187-014-0712-0.

Samala, R. V., Hoeksema, L. J., \& Colbert, C. Y. (2019). A Qualitative Study of Independent Home Visits by Hospice Fellows: Addressing Gaps in ACGME Milestones by Fostering Reflection and Self-Assessment.Am J Hosp Palliat Care. Oct;36(10):885-92. doi.org/10.1177\%2F1049909119836218

Saylor, J., Vernoony, S., Selekman, J., \& Cowperthwait, A. (2016). Interprofessional Education Using a Palliative Care Simulation. Nurse Educ. MayJun;41(3):125-29. 10.1097/NNE.0000000000000228. 
Schaeffer, K. et al. (2014). Raising the bar for the seriously III patients: Results of a National survey to define essential competencies of medical students and residents. NIH - Public access. Author manuscript. Acad Med. July;89(7): 1024-31. 10.1097/ACM.0000000000000271

Schiessl, C., Walshe, M., Wildfeuer, S., Larkin, P., Voltz, R., \& Juenger, J. (2013). Undergraduate curricula in palliative medicine: a systematic analysis based on the palliative education assessment tool. J Palliat Med; 16(1): 20-30. 10.1089/jpm.2012.0281.

Schillerstrom, J. E.,Sanchez-Reilly, S., \& O'Donnell, L.(2012).Improving student comfort with death and dying discussions through facilitated family encounters. Acad Psychiatry, 36(3): 188-90. 10.1176/appi.ap.10020032.

Schneidernam, L. J. (2011) Defining Medical Futility and Improving Medical Care. Journal of Bioethical Inquiry,8:123-31. 10.1007/s11673-011-9293-3.

Shaheen, A., Denton, G. D., Stratton, T. D., \& Hoellein, A. R.(2014). Chretien KC End-of-life and palliative care curricula in internal medicine clerkships: a report on the presence, value, and design of curricula as rated by clerkship directors Acad Med; 2014, 89(8): 1168-73. 10.1097/ACM.0000000000000311.

Smith, A., Carey, C., Sadler, J., Smith, H., Stephens, R., \& Frith, C. (2019). Undergraduate education in anaesthesia, intensive care, pain, and perioperative medicine: The development of a national curriculum framework. Med Teach. Mar;41(3):340-46. 10.1080/0142159X.2018.1472373.

Smith, G. M., \& Schaefer, K. G. (2014).Missed opportunities to train medical students in generalist palliative care during core clerkships. J Palliat Med; 17(12): 1344-7.

Schulz-Quach, C., Wenzel-Meyburg, U., \& Fetz, K. (2018). Can elearning be used to teach palliative care? - medical students' acceptance,knowledge, and selfestimation of competence in palliative care after elearning. BMC Med Educ. Apr 27;18-82. 10.1186/s12909-018-1186-2

Spalding, J., \&Yardley, S. (2016). 'The nice thing about doctors is that you can sometimes get a day off school': anaction research study to bring lived experiences from children, parents and hospice staff into medical students' preparation for practice BMJ Support Palliat Care. Dec;6(4):459-64. 10.1136/bmjopen-201601389

Taquete, S. R., \& Rego, S. et all, (2005) Situações conflituosas vivenciadas por estudantes de medicina. Rev Assoc Med Bras; 51(1): 23-28. ISSN 18069282. https://doi.org/10.1590/S0104-42302005000100015.

Taquete, S. R., \& Borges, L. M. (2020). Pesquisa qualitativa para todos: Vozes..

Vergo, M. T., Sachs, S., MacMartin, M. A., Kirkland, K. B., Cullinan, A. M., \& Stephens, L. A. (2017). Acceptability and Impact of a Required Palliative Care Rotation with Prerotation and Postrotation Observed Simulated Clinical Experience during Internal Medicine Residency Training on Primary Palliative Communication Skills. J Palliat Med. May ;20(5):542-47. 10.1089/jpm.2016.0348

Von Gunten, F., Mullan, P. B., Nelesen, R., Garman, K., McNeal, H., Savoia, M., et all. (2017). Primary Care Residents Improve Knowledge, Skills, Attitudes, and Practice After aClinical Curriculum With a Hospice. Am J Hosp Palliat Care. Sep;34(8):713-20. 10.1177/1049909116655767

Weber, M.,Schmiedel, S., Nauck, F., \& Alt-Epping, B. (2016).Knowledge and attitude of medical students in Germany towards palliative care : Does the final year of medical school make a difference?]. Schmerz. Jun;30(3):279-85. 10.1007/s00482-015-0055-8

World Health Organization.(2002). National cancer control programmes: policies and managerial guidelines, (2nd ed.) Geneva: World Health Organization, books.google.com. https://www.who.int/cancer/publications/nccp2002/en/

World Health Organization. (2014). Global Atlas of Palliative Care at the end-of-life.http://www.who.int/nmh/Global_Atlas_of_Palliative_Care.pdf 